\title{
Borsuk-Sieklucki theorem in cohomological dimension theory
}

by

\begin{abstract}
Margareta Boege (Cuernavaca), Jerzy Dydak (Knoxville, TN), Rolando Jiménez (Cuernavaca), Akira Koyama (Osaka) and Evgeny V. Shchepin (Moscow)
\end{abstract}

\begin{abstract}
The Borsuk-Sieklucki theorem says that for every uncountable family $\left\{X_{\alpha}\right\}_{\alpha \in A}$ of $n$-dimensional closed subsets of an $n$-dimensional ANR-compactum, there exist $\alpha \neq \beta$ such that $\operatorname{dim}\left(X_{\alpha} \cap X_{\beta}\right)=n$. In this paper we show a cohomological version of that theorem:

TheOREM. Suppose a compactum $X$ is $\operatorname{clc}_{\mathbb{Z}}^{n+1}$, where $n \geq 1$, and $G$ is an Abelian group . Let $\left\{X_{\alpha}\right\}_{\alpha \in J}$ be an uncountable family of closed subsets of $X$. If $\operatorname{dim}_{G} X=\operatorname{dim}_{G} X_{\alpha}=n$ for all $\alpha \in J$, then $\operatorname{dim}_{G}\left(X_{\alpha} \cap X_{\beta}\right)=n$ for some $\alpha \neq \beta$.

For $G$ being a countable principal ideal domain the above result was proved by Choi and Kozlowski [C-K]. Independently, Dydak and Koyama [D-K] proved it for $G$ being an arbitrary principal ideal domain and posed the question of validity of the Theorem for quasicyclic groups (see Problem 1 in $[\mathrm{D}-\mathrm{K}]$ ).

As applications of the Theorem we investigate equality of cohomological dimension and strong cohomological dimension, and give a characterization of cohomological dimension in terms of a special base.
\end{abstract}

1. Introduction. Borsuk $[\mathrm{Bo}]$ and Sieklucki $[\mathrm{S}]$ investigated dimension properties of ANR-compacta and proved the following:

1.1. TheOREM. Let $\left\{X_{\alpha}\right\}_{\alpha \in A}$ be an uncountable family of $n$-dimensional closed subsets of an $n$-dimensional ANR-compactum. Then there exist $\alpha \neq \beta$ in $A$ such that $\operatorname{dim}\left(X_{\alpha} \cap X_{\beta}\right)=n$.

2000 Mathematics Subject Classification: 55M10, 54F45.

Key words and phrases: cohomological dimension, cohomology locally $n$-connected compacta, ANR, descending chain condition.

The second author is partially supported by grant DMS-0072356 from the National Science Foundation.

The third author is partially supported by CONACYT (25314E).

The fifth author is partially supported by Russian Foundation of Basic Research (99-01-00009) and CONACYT (980066). 
It is easy to see that Theorem 1.1 holds for $X$ being the $n$-cube. Indeed, the interiors $\operatorname{int}\left(X_{\alpha}\right)$ must be nonempty, so there is a pair of indices $\alpha \neq \beta$ so that $\operatorname{int}\left(X_{\alpha} \cap X_{\beta}\right) \neq \emptyset$. Hence all $n$-dimensional manifolds and $n$-dimensional polyhedra have the same property. On the other hand, an $n$-dimensional compactum may admit an uncountable family $\left\{X_{\alpha}\right\}_{\alpha \in A}$ of $n$-dimensional (closed) subsets such that $\operatorname{dim}\left(X_{\alpha} \cap X_{\beta}\right) \leq n-1$ for all $\alpha \neq \beta$ in $A$. For example, the $n$-dimensional Menger compactum $\mu^{n}, n \geq 1$, contains a copy of the product of the $n$-disk $D^{n}$ and the Cantor set $C$. Thus, $\mu^{n}$ contains an uncountable family of pairwise disjoint $n$-dimensional closed subsets.

Choi and Kozlowski [C-K] generalized the Borsuk-Sieklucki theorem by using cohomological local connectivity and cohomological dimension based on Alexander-Spanier cohomology with compact supports and with coefficients in a countable principal ideal domain $R$. Their theorem states that if $X$ is a clc ${ }^{n}$ locally compact separable metric space with $\operatorname{dim}_{R} X=n$ and $\left\{X_{\lambda}\right\}_{\lambda \in \Lambda}$ is an uncountable collection of closed subsets of $X$ with $\operatorname{dim}_{R} X_{\lambda}=n$ for all $\lambda$, then there are two distinct indices $\mu, \lambda \in \Lambda$ such that $\operatorname{dim}_{R}\left(X_{\mu} \cap X_{\lambda}\right)=n$.

Independently, Dydak and Koyama [D-K] undertook an effort to generalize the Borsuk-Sieklucki theorem to cohomological dimension. Here is their result:

1.2. Theorem. Suppose that a compactum $X$ is $\operatorname{clc}_{R}^{n}$, where $n \geq 1$ and $R$ is a principal ideal domain. Let $\left\{X_{\alpha}\right\}_{\alpha \in A}$ be an uncountable family of closed subsets of $X$. If $\operatorname{dim}_{R} X=n$ and $\operatorname{dim}_{R} X_{\alpha}=n$ for each $\alpha \in A$, then there is a pair of indices $\alpha \neq \beta$ in $A$ such that $\operatorname{dim}_{R}\left(X_{\alpha} \cap X_{\beta}\right)=n$.

Thus, the result of $[\mathrm{D}-\mathrm{K}]$ drops the assumption of $R$ being countable and weakens the connectivity of $X$. However, the proof in $[\mathrm{C}-\mathrm{K}]$ is more elegant. The virtue of the proof in $[\mathrm{D}-\mathrm{K}]$ is that its idea can be applied to arbitrary groups, which is the goal of this paper. We prove a variant of Theorem 1.2 in which the principal ideal domain $R$ is replaced by a group satisfying the descending chain condition. This allows us to deduce a generalization of the Borsuk-Sieklucki theorem for arbitrary groups if $X$ is locally $(n+1)$-connected.

As an application, we investigate equality of cohomological dimension and strong cohomological dimension introduced by Kodama $[\mathrm{K}]$. Also, we give a characterization of cohomological dimension in terms of existence of a special base.

2. Preliminaries. Let $X$ be a compactum. The cohomological dimension of $X$ with respect to the abelian group $G$, denoted by $\operatorname{dim}_{G} X$, is said to be less than or equal to $n$ (notation: $\operatorname{dim}_{G} X \leq n$ ) provided that 
$H^{k}(X, A ; G)=0$ for all $k \geq n+1$ and all closed subsets $A \subset X$, where $H^{*}$ is the Cech cohomology theory (see $\left[\mathrm{Dr}_{1,2}\right],[\mathrm{K}]$, or $[\mathrm{Ku}]$ ).

We are interested in the cohomological dimension where the coefficient group is the quasicyclic group $\mathbb{Q}_{p}$ (sometimes also denoted by $\mathbb{Z}\left(p^{\infty}\right)$ ) defined as follows:

Let $p$ be a fixed prime number. The quasicyclic group $\mathbb{Q}_{p}$ is the union of all $p^{k}$ th roots of unity, where $k$ ranges over all positive integers. There is another way of describing $\mathbb{Q}_{p}$ : for $n \in \mathbb{N}$ define $\mathbb{Z}_{1 / p^{n}}=\left\{m / p^{n}: m\right.$ is an integer modulo $\left.p^{n}\right\}$. Then $\mathbb{Q}_{p}$ is the set $\bigcup_{n=1}^{\infty} \mathbb{Z}_{1 / p^{n}}$ together with the abelian group operation $+: \mathbb{Q}_{p} \times \mathbb{Q}_{p} \rightarrow \mathbb{Q}_{p}$ defined by

$$
\frac{m}{p^{n}}+\frac{r}{p^{s}}=\frac{p^{k-n} m+p^{k-s} r}{p^{k}}
$$

where $k=\max \{n, s\}$ and the sum is modulo $p^{k}$. Observe that each subgroup $\mathbb{Z}_{1 / p^{n}}$ can be generated by an element of the form $m / p^{n}$ where $m$ and $p^{n}$ are relatively prime.

An alternative description of $\mathbb{Q}_{p}$ is as the quotient group $\mathbb{Q} / \mathbb{Z}_{(p)}$, where $\mathbb{Z}_{(p)}$ is the group of all rational numbers $m / n$ so that $n$ is relatively prime to $p$. Under this description $\mathbb{Z}_{1 / p^{n}}$ is the image of the group of all rational numbers $m / p^{k}$, where $k \leq n$.

2.1. Definition. An abelian group satisfies the descending chain condition (see [Hun], p. 374) if every decreasing chain $B_{1} \supset B_{2} \supset \ldots$ of subgroups of $G$ stabilizes (i.e., there exists $n$ so that $B_{i}=B_{i+1}$ for all $i>n$ ).

2.2. Lemma. (1) The quasicyclic group $\mathbb{Q}_{p}$ satisfies the descending chain condition.

(2) If $G_{1}, \ldots, G_{k}$ satisfy the descending chain condition, then so does $G_{1} \oplus \ldots \oplus G_{k}$.

Proof. (1) is a consequence of the fact that all proper subgroups of $\mathbb{Q}_{p}$ are $\mathbb{Z}_{1 / p^{n}}$ and $\mathbb{Z}_{1 / p} \subset \mathbb{Z}_{1 / p^{2}} \subset \ldots$ Indeed, if $B$ is a nontrivial subgroup of $\mathbb{Q}_{p}$ and $0 \neq m / p^{n} \in B$, where $m$ and $p$ are relatively prime, then $B$ contains $\mathbb{Z}_{1 / p^{n}}$ (as $B$ is generated by $m / p^{n}$ ). If $B$ is a proper subgroup of $\mathbb{Q}_{p}$, then there is the smallest integer $k$ so that $B$ contains $\mathbb{Z}_{1 / p^{k}}$ but does not contain $\mathbb{Z}_{1 / p^{k+1}}$. If $m / p^{n} \in B-\mathbb{Z}_{1 / p^{k}}$, where $m$ and $p$ are relatively prime, then $n$ cannot be greater than $k$ as $B$ does not contain $\mathbb{Z}_{1 / p^{k+1}}$, a contradiction. Thus $B=\mathbb{Z}_{1 / p^{k}}$.

(2) is essentially Corollary 1.7 in [Hun], p. 374.

It is easy to prove that if $G$ and $H$ are groups, $G$ satisfies the descending chain condition and $f: G \rightarrow H$ is an epimorphism, then $H$ also satisfies the descending chain condition. Hence if $K$ is a subgroup of a group $G$ satisfying 
the descending chain condition then both $K$ and $G / K$ satisfy the descending chain condition.

We need to generalize the concept of pro-epimorphisms of $[\mathrm{D}-\mathrm{K}]$ to homomorphisms of groups.

2.3. Definition. Suppose $G$ is a group and $\left\{G_{\alpha}\right\}_{\alpha \in J}$ a family of groups. A homomorphism $f: G \rightarrow \prod_{\alpha \in J} G_{\alpha}$ is called a pro-epimorphism if, for any finite subset $F$ of $J$, the composition

$$
G \stackrel{f}{\longrightarrow} \prod_{\alpha \in J} G_{\alpha} \stackrel{p_{F}}{\longrightarrow} \prod_{\alpha \in F} G_{\alpha}
$$

is an epimorphism.

The following result is a version of the Basic Lemma of $[\mathrm{D}-\mathrm{K}]$ for groups satisfying the descending chain condition.

2.4. Proposition. Let $G$ be a group satisfying the descending chain condition and let $f: G \rightarrow \prod_{\alpha \in J} G_{\alpha}$ be a pro-epimorphism, where $\left\{G_{\alpha}\right\}_{\alpha \in J}$ is a family of groups. If $J$ is infinite, then there is $\beta \in J$ such that $G_{\beta}=0$.

Proof. Consider the subset $J^{\prime}=\left\{\alpha \in J \mid G_{\alpha} \neq\{0\}\right\}$ of $J$. We plan to show that $J^{\prime}$ is finite. Suppose $J^{\prime}$ is infinite and pick an infinite sequence $\alpha_{i} \in J^{\prime}, i \geq 1$, of points of $J^{\prime}$. Define $F_{k}, k \geq 1$, as $\left\{\alpha_{1}, \ldots, \alpha_{k}\right\}$. Define $A_{k}$ as the kernel of $p_{F_{n}} \circ f$ for $k \geq 1$. Since $G$ satisfies the descending chain condition, there is $n \geq 2$ so that $A_{n}=A_{n-1}$. Pick $c \in G_{\alpha_{n}}-\{0\}$. Since $p_{F_{n}} \circ f$ is an epimorphism, there is $d \in G$ so that $p_{F_{n}} \circ f(d)=(0, \ldots, 0, c)$. That means $d \in A_{n-1}-A_{n}$, a contradiction.

The next result is a modification of the Pro Lemma in $[\mathrm{D}-\mathrm{K}]$ with a finitely generated module replaced by a group satisfying the descending chain condition.

2.5. Lemma. Consider a commutative diagram of abelian groups

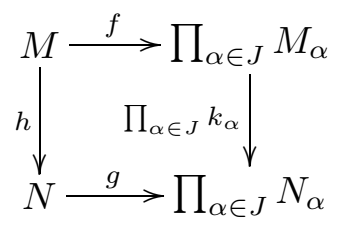

so that $f$ is a pro-epimorphism and $J$ is infinite. If $\operatorname{im}(h)$ satisfies the descending chain condition, then there is $\alpha \in J$ such that $k_{\alpha}: M_{\alpha} \rightarrow N_{\alpha}$ is trivial.

Proof. We can apply 2.4 to the homomorphism $u=g \mid \operatorname{im}(h): \operatorname{im}(h) \rightarrow$ $\prod_{\alpha \in J} \operatorname{im}\left(k_{\alpha}\right)$ by noticing that $u$ is a pro-epimorphism since $f$ is, and the diagram $(*)$ is commutative. 
The following lemma is a version of Lemma 17.3 of [Br] for groups satisfying the descending chain condition.

2.6. Lemma. Consider the commutative diagram of abelian groups

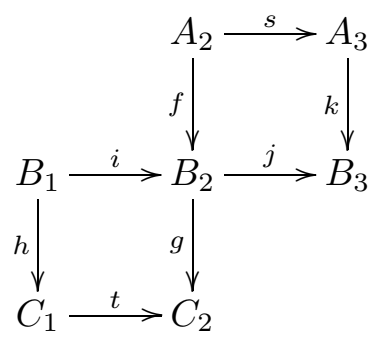

in which the middle row is exact. If both $\operatorname{im}(h)$ and $\operatorname{im}(k)$ satisfy the descending chain condition, then so does $\operatorname{im}(g \circ f)$.

Proof. Suppose there is an infinite decreasing sequence $A_{1} \supset A_{2} \supset \ldots$ of subgroups of $\operatorname{im}(g \circ f) \subset C_{2}$. Since $\operatorname{im}(h)$ satisfies the descending chain condition, the chain $\operatorname{im}(t \circ h) \cap A_{i}$ stabilizes. Without loss of generality, we may assume $\operatorname{im}(t \circ h) \cap A_{i}=\operatorname{im}(t \circ h) \cap A_{j}$ for all $i, j$. Since $\operatorname{im}(k)$ satisfies the descending chain condition, the chain $(k \circ s)(g \circ f)^{-1}\left(A_{i}\right)$ stabilizes. Again, we may assume $(k \circ s)(g \circ f)^{-1}\left(A_{i}\right)=(k \circ s)(g \circ f)^{-1}\left(A_{j}\right)$ for all $i, j$. This is the same as $j\left(g^{-1}\right)\left(A_{i}\right)=j\left(g^{-1}\right)\left(A_{j}\right)$ for all $i, j$. Suppose $x \in A_{i}-A_{j}$ for some $j>i$. Pick $x^{\prime} \in B_{2}$ so that $g\left(x^{\prime}\right)=x$. There is $y^{\prime} \in g^{-1}\left(A_{j}\right)$ with $j\left(y^{\prime}\right)=j\left(x^{\prime}\right)$. Pick $z^{\prime} \in B_{1}$ with $i\left(z^{\prime}\right)=x^{\prime}-y^{\prime}$. Notice that $t\left(h\left(z^{\prime}\right)\right) \in A_{i}$, so there is $z^{\prime \prime} \in i^{-1}\left(g^{-1}\left(A_{j}\right)\right)$ with $t\left(h\left(z^{\prime}\right)\right)=t\left(h\left(z^{\prime \prime}\right)\right)$. This implies $g\left(i\left(z^{\prime}\right)\right)=$ $g\left(x^{\prime}\right)-g\left(y^{\prime}\right)=g\left(i\left(z^{\prime \prime}\right)\right) \in A_{j}$. Thus $x=g\left(x^{\prime}\right)=\left(g\left(x^{\prime}\right)-g\left(y^{\prime}\right)\right)+g\left(y^{\prime}\right) \in A_{j}$, a contradiction.

2.7. Definition. A compactum $X$ is said to be cohomology locally $n$ connected with respect to an abelian group $G$ ( $X$ is $n$ - clc $G$ for short) if for each point $x \in X$ and neighborhood $N$ of $x$, there is a neighborhood $M \subset N$ of $x$ such that the inclusion-induced homomorphism

$$
i_{M, N}^{n}: H^{n}(N ; G) \rightarrow H^{n}(M ; G)
$$

is trivial, where $H^{*}$ is the reduced Cech cohomology theory.

$X$ is said to be $\operatorname{clc}_{G}^{n}$ if it is $k-\operatorname{clc}_{G}$ for all $k \leq n$.

2.8. Theorem. Suppose $G$ is an Abelian group satisfying the descending chain condition. If $X$ is $\operatorname{clc}_{G}^{n}$, and $K$ and $L$ are closed subsets of $X$ with $K \subset \operatorname{int}(L)$, then the image of the inclusion-induced homomorphism $i_{K, L}^{k}$ : $H^{k}(L ; G) \rightarrow H^{k}(K ; G)$ satisfies the descending chain condition for $k=$ $0, \ldots, n$.

Proof. First consider the image of $i_{K, L}^{0}$. As $X$ is $\operatorname{clc}_{G}^{0}$ and $K$ is compact, $K$ is contained in the union of finitely many components of $L$. This means 
that $H^{0}(L ; G) \rightarrow H^{0}(K ; G)$ factors through a finite direct sum of copies of $G$. By Lemma $2.2, \operatorname{im}\left(i_{K, L}^{0}\right)$ satisfies the descending chain condition.

Suppose 2.8 holds for all $n<m, X$ is $\operatorname{clc}_{G}^{m}$, and $K$ and $L$ are closed subsets of $X$ with $K \subset \operatorname{int}(L)$. Consider the family $\mathcal{F}$ of all compact subsets $C$ of the interior of $L$ so that the image of the inclusion-induced homomorphism $i_{C^{\prime}, L}^{m}: H^{m}(L ; G) \rightarrow H^{m}\left(C^{\prime} ; G\right)$ satisfies the descending chain condition for some compact neighborhood $C^{\prime}$ of $C \operatorname{in} \operatorname{int}(L)$. Since $X$ is $\operatorname{clc}_{G}^{m}$, each point $x \in \operatorname{int}(L)$ has a compact neighborhood $C_{x} \in \mathcal{F}$. If we prove that $\mathcal{F}$ is closed under taking unions of sets, we are done as $K$ can be covered by a finite union of elements of $\mathcal{F}$. Suppose $C_{1}, C_{2} \in \mathcal{F}$. Pick a neighborhood $C_{j}^{\prime}$ of $C_{j}$ in $\operatorname{int}(L)$ so that the image of the inclusion-induced homomorphism $i_{C_{j}^{\prime}, L}^{m}: H^{m}(L ; G) \rightarrow H^{m}\left(C_{j}^{\prime} ; G\right)$ satisfies the descending chain condition for $j=1,2$. Pick a neighborhood $C_{j}^{\prime \prime}$ of $C_{j}$ in $\operatorname{int}\left(C_{j}^{\prime}\right)$ for $j=1,2$. Applying the Meyer-Vietoris exact sequence and Lemma 2.6 to the following diagram shows that $\operatorname{im}\left(i_{C_{1}^{\prime \prime} \cup C_{2}^{\prime \prime}, L}^{m}\right)$ satisfies the descending chain condition:

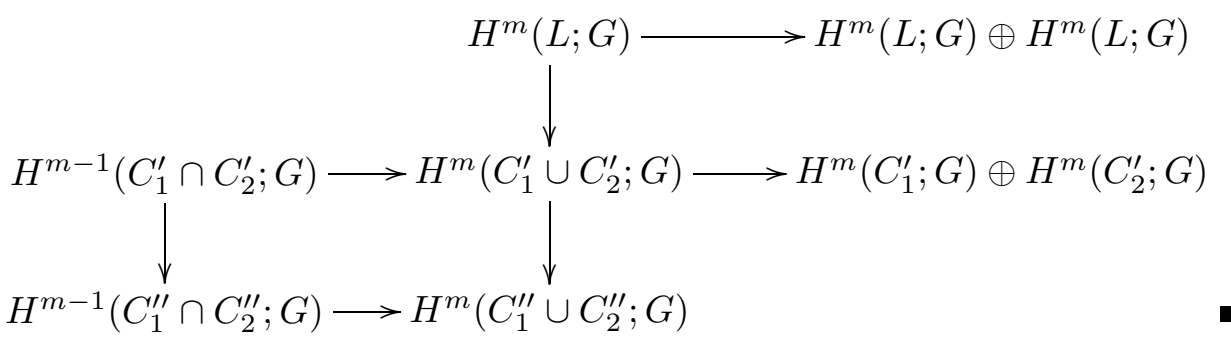

The next result is an analog of Theorem 2.2 in $[\mathrm{D}-\mathrm{K}]$ with a finitely generated module replaced by a group satisfying the descending chain condition.

2.9. Theorem. Suppose $G$ is an Abelian group satisfying the descending chain condition. If $X$ is $\operatorname{clc}_{G}^{n}$ and $A_{1}, A_{2}, B_{1}, B_{2}$ are closed subsets of $X$ such that $A_{1} \subset B_{1}, A_{2} \subset B_{2}, A_{1} \subset \operatorname{int}\left(A_{2}\right)$ and $B_{1} \subset \operatorname{int}\left(B_{2}\right)$, then the image of $H^{k}\left(B_{2}, A_{2} ; G\right) \rightarrow H^{k}\left(B_{1}, A_{1} ; G\right)$ satisfies the descending chain condition for all $k \leq n$.

Proof. Use induction and apply Lemma 2.6 to the commutative diagram

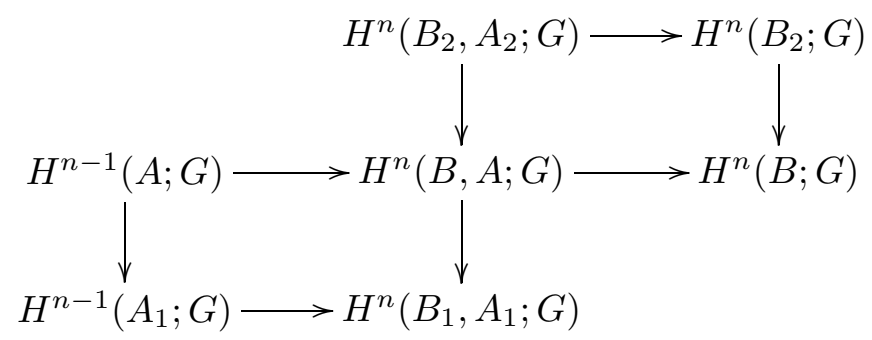

where $A=A_{1} \cup A_{2}$ and $B=B_{1} \cup B_{2}$. 
3. Borsuk-Sieklucki theorem. Using our previous results we are ready to prove the Borsuk-Sieklucki theorem for groups satisfying the descending chain condition in an analogous way to that of Dydak and Koyama $[\mathrm{D}-\mathrm{K}]$ for rings.

3.1. Theorem. Suppose a compactum $X$ is $\operatorname{clc}_{G}^{n}$, where $n \geq 1$, and $G$ is an Abelian group satisfying the descending chain condition. Let $\left\{X_{\alpha}\right\}_{\alpha \in J}$ be an uncountable family of closed subsets of $X$. If $\operatorname{dim}_{G} X=\operatorname{dim}_{G} X_{\alpha}=n$ for all $\alpha \in J$, then $\operatorname{dim}_{G}\left(X_{\alpha} \cap X_{\beta}\right)=n$ for some $\alpha \neq \beta$.

Proof. Suppose that $\operatorname{dim}_{G}\left(X_{\alpha} \cap X_{\beta}\right) \leq n-1$ for each pair $\alpha \neq \beta$ in $J$. For each $\alpha \in J$, there is a closed subset $C_{\alpha} \subset X_{\alpha}$ such that $H^{n}\left(X_{\alpha}, C_{\alpha} ; G\right) \neq 0$, because $\operatorname{dim}_{G} X_{\alpha}=n$. Let $\left\{N_{i}\right\}_{i=1}^{\infty}$ be a countable family of closed subsets of $X$ such that for each closed subset $K$ of $X$ the family $\left\{N_{i}: K \subset \operatorname{int}\left(N_{i}\right)\right\}$ is a basis of neighborhoods of $K$ in $X$. By the continuity of Cech cohomology, for each $\alpha \in J$, there is $N_{k(\alpha)} \subset \operatorname{int}\left(N_{h(\alpha)}\right)$ such that the inclusion-induced homomorphism

$$
H^{n}\left(X_{\alpha}, X_{\alpha} \cap N_{h(\alpha)} ; G\right) \rightarrow H^{n}\left(X_{\alpha}, X_{\alpha} \cap N_{k(\alpha)} ; G\right)
$$

is not trivial.

Since $J$ is uncountable, we may assume that $N_{k(\alpha)}=N$ for every $\alpha \in J$, and $N_{h(\alpha)}=M$ for every $\alpha \in J$. Namely, we have closed subsets $M, N$ of $X$ such that $N \subset \operatorname{int}(M)$ and for every $\alpha \in J$,

$$
H^{n}\left(X_{\alpha}, X_{\alpha} \cap M ; G\right) \rightarrow H^{n}\left(X_{\alpha}, X_{\alpha} \cap N ; G\right) \text { is not trivial. }
$$

On the other hand, since $\operatorname{dim}_{G}\left(X_{\alpha} \cap X_{\beta}\right) \leq n-1$ for all pairs $\alpha \neq \beta$ in $J$,

$$
H^{n}\left(\bigcup_{\alpha \in F} X_{\alpha},\left(\bigcup_{\alpha \in F} X_{\alpha}\right) \cap M ; G\right) \rightarrow \bigoplus_{\alpha \in F} H^{n}\left(X_{\alpha}, X_{\alpha} \cap M ; G\right)
$$

is an epimorphism for all finite subsets $F \subset J$.

We note that the inclusion-induced homomorphism

$$
H^{n}\left(\left(\bigcup_{\alpha \in F} X_{\alpha}\right) \cup M, M ; G\right) \rightarrow H^{n}\left(\bigcup_{\alpha \in F} X_{\alpha},\left(\bigcup_{\alpha \in F} X_{\alpha}\right) \cap M ; G\right)
$$

is an isomorphism by the excision axiom, and the inclusion-induced homomorphism

$$
H^{n}(X, M ; G) \rightarrow H^{n}\left(\left(\bigcup_{\alpha \in F} X_{\alpha}\right) \cup M, M ; G\right)
$$

is an epimorphism because $\operatorname{dim}_{G} X=n$. Hence, for all finite subsets $F \subset J$,

$$
H^{n}(X, M ; G) \rightarrow \bigoplus_{\alpha \in F} H^{n}\left(X_{\alpha}, X_{\alpha} \cap M ; G\right) \quad \text { is an epimorphism. }
$$


Now we consider the following commutative diagram:

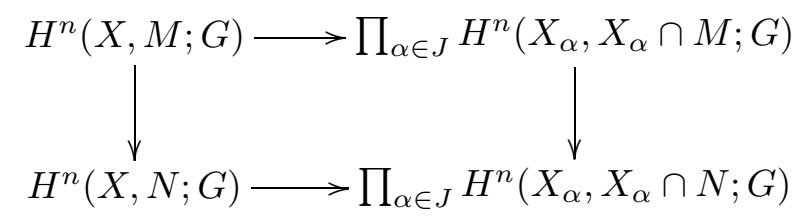

Property (2) says that the upper homomorphism is a pro-epimorphism. By Theorem 2.8, the image of $H^{n}(X, M ; G) \rightarrow H^{n}(X, N ; G)$ satisfies the descending chain condition. Hence, by 2.5 , there exists $\alpha \in J$ such that $H^{n}\left(X_{\alpha}, X_{\alpha} \cap M ; G\right) \rightarrow H^{n}\left(X_{\alpha}, X_{\alpha} \cap N ; G\right)$ is trivial. This contradicts (1). Therefore there are $\alpha \neq \beta$ in $J$ such that $\operatorname{dim}_{G}\left(X_{\alpha} \cap X_{\beta}\right)=n$.

3.2. TheOREM. Suppose a compactum $X$ is $\operatorname{clc}_{\mathbb{Z}}^{n+1}$ and $G$ is an Abelian group, where $n \geq 1$. Let $\left\{X_{\alpha}\right\}_{\alpha \in J}$ be an uncountable family of closed subsets of $X$. If $\operatorname{dim}_{G} X=\operatorname{dim}_{G} X_{\alpha}=n$ for all $\alpha \in J$, then $\operatorname{dim}_{G}\left(X_{\alpha} \cap X_{\beta}\right)=n$ for some $\alpha \neq \beta$.

Proof. Bockstein's First Theorem (see $[\mathrm{Ku}]$ ) says that there is a subset $\sigma(G)$ (we call it the Bockstein basis of $G$ ) of the family of groups consisting of $\mathbb{Q}, \mathbb{Z} / p$ ( $p$ prime), $\mathbb{Z}_{(p)}$ ( $p$ prime), and $\mathbb{Q}_{p}$ ( $p$ prime) such that $\operatorname{dim}_{G} Y=$ $\max \left\{\operatorname{dim}_{H} Y: H \in \sigma(G)\right\}$ for all $Y$ compact. Hence there is $R \in \sigma(G)$ such that $\operatorname{dim}_{R} X=n, \operatorname{dim}_{R} X_{\alpha}=n$ for uncountably many $\alpha \in J$, and $R$ is either a countable PID or a group satisfying the descending chain condition. From $X \in \operatorname{clc}_{\mathbb{Z}}^{n+1}$ the Universal Coefficient Theorem implies $X \in \operatorname{clc}_{R}^{n}$. Therefore, by Theorem 3.1 and $[\mathrm{D}-\mathrm{K}]$, there are $\alpha \neq \beta$ in $J$ such that $\operatorname{dim}_{R}\left(X_{\alpha} \cap X_{\beta}\right)=n$. In particular, $\operatorname{dim}_{G}\left(X_{\alpha} \cap X_{\beta}\right)=n$.

4. Applications. First we give applications of the cohomological dimension versions of the Borsuk-Sieklucki theorem to strong cohomological dimension introduced by Kodama $[\mathrm{K}]$ for compacta but which can be generalized to metrizable spaces without any problem.

4.1. Definition. $X$ has strong cohomological dimension at most $n$ with respect to an abelian group $G$, written $\operatorname{Ind}_{G} X \leq n$, provided that for any pair of a closed subset $A \subset X$ and an open subset $U$ containing $A$, there exists an open subset $V$ such that

$$
A \subset V \subset U \text { and } \operatorname{dim}_{G} \partial V \leq n-1 .
$$

We define

$$
\operatorname{Ind}_{G} X=\min \left\{n: \operatorname{Ind}_{G} X \leq n\right\} .
$$

Kodama proved ([K], Lemma 38-9) that for every 2-dimensional compact ANR $X$ and every nontrivial abelian group $G$, we have the equality $\operatorname{dim}_{G} X=\operatorname{Ind}_{G} X=\operatorname{dim} X=2$, and asked ([K], Problem 38-10): If $X$ is 
a compact $A N R$, does the equality $\operatorname{dim}_{G} X=\operatorname{Ind}_{G} X$ hold for every abelian group $G$ ?

This problem was affirmatively answered by Dydak and Koyama [D-K].

In this section we will improve some of the results of $[\mathrm{D}-\mathrm{K}]$ related to the strong cohomological dimension.

4.2. Theorem. Let $G$ be an abelian group. Suppose that a compactum $X$ is $\operatorname{clc}_{\mathbb{Z}}^{n+1}$. If $\operatorname{dim}_{G} X=n$, then $\operatorname{Ind}_{G} X=n$.

Proof. By Lemma 4.2 of $[\mathrm{D}-\mathrm{K}]$, it suffices to show that $\operatorname{Ind}_{G} X \leq n$. For a given closed subset $A$ of $X$, let

$$
B(A, \varepsilon)=\{x \in X: d(a, A) \leq \varepsilon\} \quad \text { and } \quad C_{\varepsilon}=\partial B(A, \varepsilon), \quad \varepsilon>0 .
$$

Since $C_{\varepsilon} \cap C_{\delta}=\emptyset$ for $\varepsilon \neq \delta$, Theorem 3.2 says that $\operatorname{dim}_{G} C_{\varepsilon}=n$ for only countably many $\varepsilon>0$. Hence $A$ has a neighborhood basis $\left\{U_{i}\right\}_{i \geq 1}$ such that $\operatorname{dim}_{G} \partial U_{i} \leq n-1$ for every $i \geq 1$. It follows that $\operatorname{Ind}_{G} X \leq n$.

Remark. Theorem 4.2 improves Theorem 4.4 of [D-K].

Now we are ready to characterize $\operatorname{dim}_{G}$ by using bases, which corresponds to well known facts in usual dimension theory.

4.3. Theorem. Suppose that $G$ is an abelian group and a compactum $X$ is $\operatorname{clc}_{\mathbb{Z}}^{n+1}$. Then the following statements are equivalent:

(i) $\operatorname{dim}_{G} X \leq n$,

(ii) $X$ has a countable base $\mathcal{B}$ such that $\operatorname{dim}_{G} \partial U \leq n-1$ for all $U \in \mathcal{B}$.

Proof. The implication (i) $\Rightarrow$ (ii) follows from Theorem 4.2. To prove that (ii) $\Rightarrow$ (i) we take a pair $A, B$ of disjoint closed subsets of $X$. Then there exist elements $U_{1}, \ldots, U_{k} \in \mathcal{B}$ such that

$$
A \subset \bigcup_{i=1}^{k} U_{i} \subset \bigcup_{i=1}^{k} \operatorname{cl}\left(U_{i}\right) \subset X \backslash B .
$$

Then the boundary $\partial\left(\bigcup_{i=1}^{k} U_{i}\right)$ separates $A$ and $B$ and $\operatorname{dim}_{G} \partial\left(\bigcup_{i=1}^{k} U_{i}\right) \leq$ $n-1$. Hence, by Lemma 4.2 of $[\mathrm{D}-\mathrm{K}], \operatorname{dim}_{G} X \leq n$.

Remark. Theorem 4.3 improves Theorem 4.6 of [D-K].

\section{References}

[Bo] K. Borsuk, Concerning the dimension of ANR-sets, Bull. Acad. Polon. Sci. 9 (1961), 685-687.

[Br] G. E. Bredon, Sheaf Theory, 2nd ed., Springer, Berlin, 1997.

[C-K] J. S. Choi and G. Kozlowski, A generalization of Sieklucki's theorem, Topology Proc. 23 (1998), 135-142. 
[Dr 1 A. N. Dranishnikov, Homological dimension theory, Russian Math. Surveys 43 (1988), no. 4, 11-63.

$\left[\mathrm{Dr}_{2}\right]-$ Basic elements of the cohomological dimension theory of compact metric spaces, preprint, 1998.

[D-K] J. Dydak and A. Koyama, Cohomological dimension of locally connected compacta, Topology Appl. 113 (2001), 39-50.

[Hun] T. W. Hungerford, Algebra, Springer, New York, 1974.

[K] Y. Kodama, Cohomological dimension theory, appendix in: K. Nagami, Dimension Theory, Academic Press, New York, 1970.

[Ku] V. I. Kuz'minov, Homological dimension theory, Russian Math. Surveys 23 (1968), no. $5,1-45$.

[S] K. Sieklucki, A generalization of a theorem of K. Borsuk concerning the dimension of ANR-sets, Bull. Acad. Polon. Sci. 10 (1962), 433-436; correction, ibid. 12 (1964), 695.

Instituto de Matemáticas

UNAM

Av. Universidad S/N, Col. Lomas de Chamilpa 62210 Cuernavaca, Morelos, México

E-mail: margaret@matcuer.unam.mx rolando@matcuer.unam.mx

Department of Mathematics

University of Tennessee

Knoxville, TN 37996, U.S.A.

E-mail: dydak@math.utk.edu
Division of Mathematical Sciences

Osaka Kyoiku University

Kashiwara, Osaka 582-8582, Japan E-mail: koyama@cc.osaka-kyoiku.ac.jp

Steklov Institute of Mathematics

Gubkina 8

117966 Moscow GSP-1, Russia

E-mail: scepin@mi.ras.ru

Received 30 March 2000;

in revised form 11 June 2001 